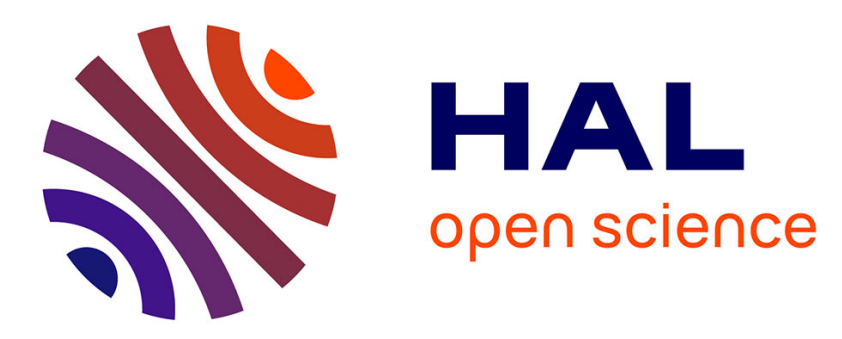

\title{
Evaluation of the effectiveness of the interval computation method to simulate the dynamic behavior of subdefinite system: application on an active suspension system
}

\author{
Hassen Trabelsi, Pierre-Alain Yvars, Jamel Louati, Mohamed Haddar
}

\section{To cite this version:}

Hassen Trabelsi, Pierre-Alain Yvars, Jamel Louati, Mohamed Haddar. Evaluation of the effectiveness of the interval computation method to simulate the dynamic behavior of subdefinite system: application on an active suspension system. International Journal on Interactive Design and Manufacturing, 2015, 9 (2), pp.83 - 96. 10.1007/s12008-014-0210-y . hal-01912051

\section{HAL Id: hal-01912051 \\ https://hal.science/hal-01912051}

Submitted on 6 Dec 2019

HAL is a multi-disciplinary open access archive for the deposit and dissemination of scientific research documents, whether they are published or not. The documents may come from teaching and research institutions in France or abroad, or from public or private research centers.
L'archive ouverte pluridisciplinaire HAL, est destinée au dépôt et à la diffusion de documents scientifiques de niveau recherche, publiés ou non, émanant des établissements d'enseignement et de recherche français ou étrangers, des laboratoires publics ou privés. 


\title{
Evaluation of the effectiveness of the interval computation method to simulate the dynamic behavior of subdefinite system: application on an active suspension system
}

\author{
Hassen Trabelsi • Pierre-Alain Yvars - Jamel Louati • \\ Mohamed Haddar
}

\begin{abstract}
A new design approach based on methods by intervals adapted to the integration of the simulation step at the earliest stage of preliminary design for dynamic systems is proposed in this study. The main idea consists on using the interval computation method to make a simulation by intervals in order to minimize the number of simulations which allow obtaining a set of solutions instead of a single one. These intervals represent the domains of possible values for the design parameters of the subdefinite system. So the parameterized model of the system is solved by interval. This avoids launching $n$ simulations with $n$ values for each design parameter. The proposed method is evaluated by several tests on a scalable numerical example. It has been applied to solve parameterized differential equations of a Macpherson suspension system and to study its dynamic behavior in its passive and active form. The dynamic model of the active suspension is nonlinear but linearisable. It is transformed into a parameterized state equation by intervals. The solution to this state equation is given in the form of a matrix exponential. Three digital implementations of exponential have been tested to obtain convergent results. Simulations results are presented and discussed.
\end{abstract}

Keywords Interval based simulation - Ordinary differential equation $\cdot$ MacPherson suspension system $\cdot$ Dynamic simulation

H. Trabelsi · P.-A.Yvars $(\varangle)$

LISMMA, Institut Supérieur de Mécanique de Paris (SupMeca), 3 rue Fernand Hainaut, 93407 Saint Ouen Cedex, France

e-mail: payvars@supmeca.fr

H. Trabelsi $\cdot$ J. Louati $\cdot$ M. Haddar

U2MP, Ecole Nationale d'Ingénieurs de Sfax, Université de Sfax, BP N1173, 3038 Sfax, Tunisia

\section{Introduction}

Conventional design methodologies based on a loop "designsimulate-back at the initial stage in case of failure" appear to be increasingly obsolete. During the past years, many studies have been conducted to optimize design, more precisely at the pre-sizing step [7-9]. This step is often expensive and requires higher computation times [8-10] in order to obtain the optimal $[10,11]$ values of design parameters. In this work we propose a new design procedure with a rigorous evaluation that allows designing a subdefinite system $[12,13]$ with a less number of simulations. The new design approach is based on the Interval computation method [14-21] and technique of constraint satisfaction problem (CSP) [22-30]. This study focus just on the evaluation of the effectiveness of the interval computation method to solve differential equations (type ODE) by intervals which represents an important step of the new design approach proposed. This study is limited to the design of linear or linearisable parametric systems, which can be reformulated in the form of state equation by intervals. The intervals represent the areas of possible values of design parameters of the system. In the new design approach, simulation step is integrated into the preliminary pre-sizing phase and the stages of ordinary design is rearranged.The paper is organized as follows: in Sect. 2, the various stages of the basic design and the new design $\mathrm{p}$ rocess were quoted and compared. Section 3 is dedicated to the presentation of interval computation method as a guaranteed method that can encompass all the solutions that satisfy requirements imposed. The idea is to apply this method to compute the dynamic simulation of a subdefinite system by solving its differential equations by intervals. In Sect. 4, the problem of exponentiation of a matrix interval and method of convergence of results is presented. Section 5 details the dynamic model of the MacPherson suspension system. A simple scal- 
able model of MacPherson suspension system is presented to check the validity of the interval computation method. The Sects. 5.1 and 6 of this paper are devoted to the application of the interval computation technique on a MacPherson suspension system in passive and active case. The interval computation technique was used to solve differential equations under uncertainties which have been applied to the values of the design parameters represented as intervals. Finally, conclusions are drawn in Sect. 6.1.2.

\section{Design approach}

Currently, the system design has to take into account most aspects of several disciplines (aerodynamics, thermal, crash, vibration ...) [1,2]. It is based on the art of compromise, i.e. accommodation of a large number of constraints. This aspect involves several multidisciplinary skills in many engineering areas. These imply the need for dialogue between all the different engineering areas during the design process, to reflect properly their specifications in the constraints of optimization, and to ensure that the generated solutions fulfil all the imposed constraints. In such cases, the human reasoning alone is not enough, because it becomes difficult to find arrangements between all requirements imposed from different disciplines, and to decide how to act to improve the chosen objectives. The complexity of the problem leads naturally to the use of robust optimization techniques to determine the best solutions. Optimization studies generate the production of a large number of simulations. So it is necessary to completely automate the launch of these simulations [3].

Stages of a usual system design process are described in Fig. 1. It is based on a loop "design-simulate-back to the ini-

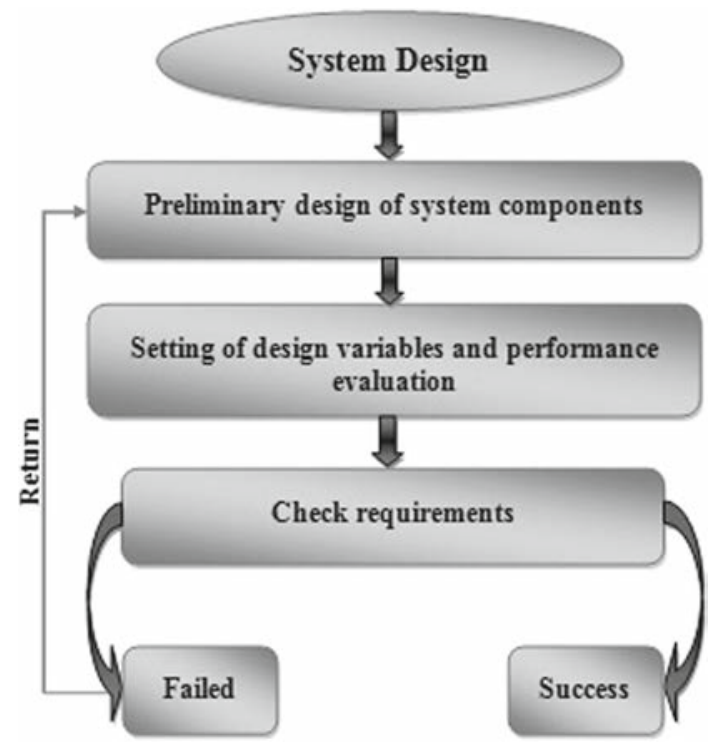

Fig. 1 General process of product design tial step in case of failure" [4]. Generally, design process is split in three main steps: the first step is to provide a preliminary design of the system components that satisfy all the requirements from a given specification. During this step, the various performance specifications that the product has to satisfy are described by a set of analytical models. Indeed, the geometry plays an important role in the preliminary design phase of the system. So in this operation the design variables of the product are defined and the dimensions are fixed. The next step is the "test". It is devoted for evaluation and simulation, allowing the designer to reduce the space of decision where solutions exist, in order to make the best choice of design variables. This step is related to the number of simulations made. Many methodologies and software tools in different engineering areas $[5,6,11]$ have been developed and applied to simulate the behaviour of a product. For example in electronics hardware description languages we find (VHDL, Verilog) which are intended to represent and simulate the behaviour and the architecture of a digital electronic system i.e. Modelica [7] for multi-physics simulation or CATIA [8] for geometry, Cosmos Works [9] to analyse dynamic behaviour of the system using finite element method for dynamic simulations. The last step of the conventional design process consists in checking the requirements. In this step the designer makes a comparison between the responses of the product obtained by simulation of the system with parameters chosen by estimation and/or by expertise and the desired response. So the designer is faced to two situations. In the first case if the resulting behaviour of the system design has satisfied the constraints imposed in the specifications document then, the design parameters used in the simulation will be taken as a solution. In the second case if the system response does not satisfy the constraints imposed, then the designer has to change these parameters by taking into account the previous simulation and the same design steps should be repeated until obtaining the optimal solution.

The previous design cannot be retained as a good approach to ensure the optimization of the system design. The designer has to go through several simulations to determine an optimal solution without being sure if the obtained solution is the global optimum within solutions space. It comes from the fact that the number of simulations that can be done is limited by the time and cost constraints which leads to oversize the system. So in order to have a robust design, we proposed through this work a new design approach in which simulation step is integrated into the preliminary design phase as shown in Fig. 2.

In Fig. 2 we present the different steps of the new design process. In this method, dynamics requirements are integrated in the preliminary design before simulation step. And among the dynamics requirements we are interested in this study to integrate dynamics simulation by intervals. 


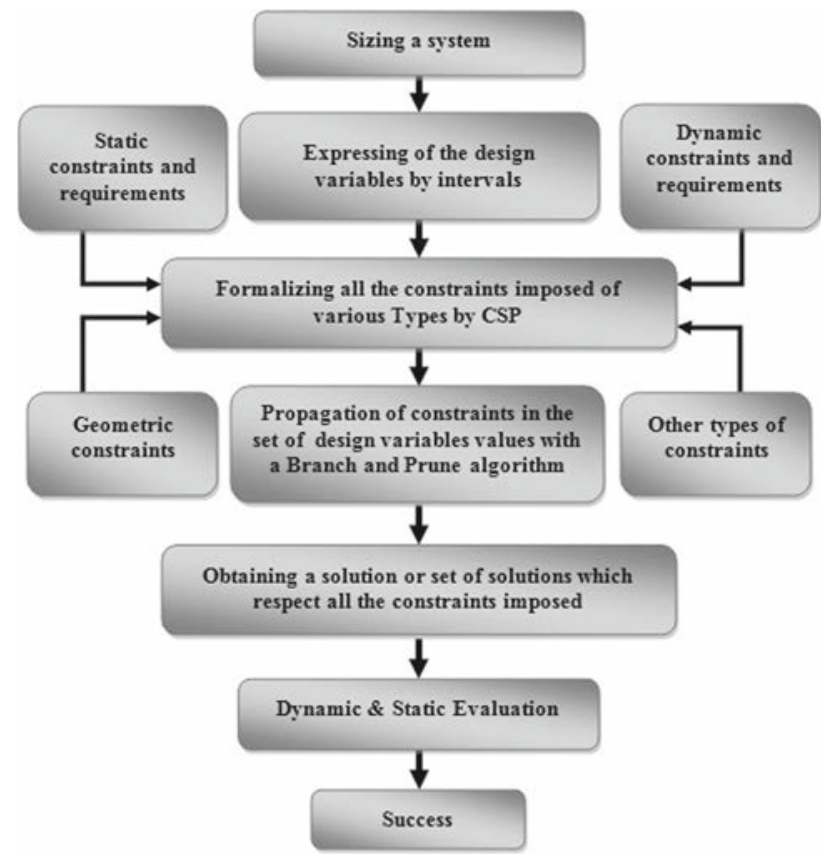

Fig. 2 Interval based design process

The new design process is made up of four main stages: the first stage is to express design variables by intervals. Here the choice of the design variables values does not require expertise. We can put a blind choice and use long intervals, but we cannot deny that expertise may reduce the computing time. In the next step, the designer identifies the requirements that must be satisfied and express these requirements as constraints on the design variables. Those constraints provide declarative descriptions of important requirements related to engineering objectives. Then all types of constraints are implemented in CSP (constraint satisfaction problem) model (in this study it is a dynamic model) in the preliminary design phase.Then the designer propagates those constraints in the intervals of design variables to frame the areas of parameter defining the system design. Here the role of CSP approach comes. In fact the CSP $[22,23]$ eliminates all values of design variables that do not respect the imposed requirements. The sets of values that remain represent the entire generated solution.

The constraint programming [22-25] is a programming paradigm that emerged in the 1980s to solve combinatorial problems of large sizes such as problems with planning and scheduling. It is a technique intended to solve the mathematical problem that looks for states or objects satisfying a number of constraints. This technique is extensively used to treat problems manipulating intervals. Indeed it serves to minimize the areas of design variables. So the idea is to apply the CSP approach to solve design problems which involve design variables that must be expressed by intervals. The CSP calculation is essentially based on the interval compu-

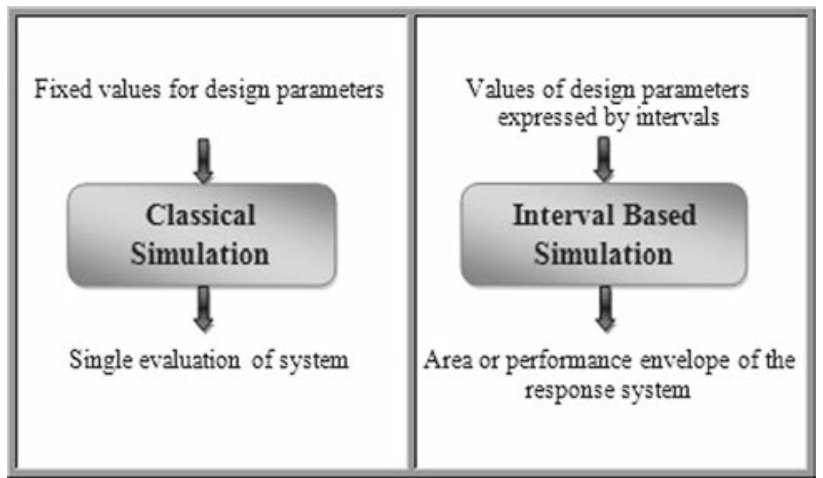

Fig. 3 Classical simulation vs. interval based simulation

tation method, so the goal of this study is to test the capability of this method to solve differential equations type (ODE).

We notice that the CSP has been extensively used in different areas of engineering including systems control $[15,19]$ and preliminary design [26-36]. In many excellent scientic works [26-36] the CSP was applied as an effective modeling language allowing the integration of requirements in the preliminary design phase. Most of requirements treated in these studies are static and/or ecological. So we propose in this work to integrate dynamics requirements such simulation by intervals of dynamic behavior of a technical system.

For the next step, the designer makes a simulation by intervals obtained by the CSP approach in order to determine the system response. Then he chooses the optimal solution from the set of solutions obtained by the interval simulation. As shown in Fig. 3 the interval based simulation method provides not only a single evaluation of the system behavior but also a set of performance bounds within the actual response system resides.

Theoretically and using what we presented before, with the new design process we can obtain the system response by interval and frame the solution space thanks to the interval computation. However the current problem, is that the evaluation of this method to solve a differential equation system and determine the dynamic response has not yet been tested on a scalable example. So the main objective of this work is to evaluate the effectiveness of the interval based simulation method to simulate the dynamic behavior of a parametric system (i.e. Macpherson suspension system) by intervals.

\section{Interval computation}

The interval computation[14-21] has been used to solve problems of uncertainty, it allows taking into account the measurement error by replacing $x$ value measured with imprecision $\varepsilon$ by the interval $[x-\varepsilon, x+\varepsilon]$. For example, replace a value not exactly representable, as $\pi$, by an interval 
containing $[3,14,3,15]$. Since the introduction of the interval computation, many applications have been developed such as linear optimization, solving ordinary differential equations, treatment of uncertainties,... The objective of the interval computation is to provide results that contain certain value or all searched values; so we talk about guaranteed or validated results. Indeed, in the interval computation, we do not handle numbers, but intervals. The result of an operation between two intervals $[18,19]: x \circledast y$, of a function $f(z)$ is the smallest interval (or vector interval) in the sense of inclusion containing:

$\{(x \circledast y \mid x \in X, y \in Y) \Longrightarrow(f(z) \mid z \in Z)\}$

Example:

$$
\begin{aligned}
& {[\underline{x} ; \bar{x}] \cdot[\underline{y} ; \bar{y}]} \\
& \quad=[\min (\underline{x} \cdot \underline{y}, \underline{x} \cdot \bar{y}, \bar{x} \cdot \underline{y}, \bar{x} \cdot \bar{y}) ; \max (\underline{x} \cdot \underline{y}, \underline{x} \cdot \bar{y}, \bar{x} \cdot \underline{y}, \bar{x} \cdot \bar{y})]
\end{aligned}
$$

The smallest interval means the smallest interval containing all possible results of the operation applied to all elements $x$ of $X$ and all elements $y$ of $Y$, all the possible results of ' $f$ ' applied to all elements $z$ of $Z$. The interval computation continues to grow, but with different objectives, for example, it represent an accurate tool to determine the global optimal or all zeros of a continuous function [16]. In this paper the objective was the application of an interval computation on a real system to get its dynamic behavior, through the resolution of its differential equations by interval.

\section{Solving linear differential equations by interval}

Solutions and methods for solving differential equations have been discussed in many papers, especially the methods that use generic intervals $[37,39]$ instead of fixed values in their calculation algorithm. Unfortunately the evaluation of those methods in real cases provided disappointing results, that often diverge. In this paper we considered the linear case hoping to get a better convergence. A linear differential equation system is defined by:

$\left\{y^{*}(t)=A . y(t)\right.$ and $y(0)=y_{0} \mid y \in \mathbb{R}^{n}$ and $\left.A \in \mathbb{R}^{n \times n}\right\}$

where $A$ is a matrix whose coefficients $a_{i i}$ that can be the design parameters of a system are expressed by intervals. The solution of this differential system is: $y(t)=\exp (t . A) \cdot y_{0}$. So the exponentiation of a real matrix can solve problems of linear ordinary differential equations (ODE) with an initial value (IVPs). The numerical calculation of the exponential of the matrix has been extensively studied $[39,40]$ to get more accurate and realistic results. Indeed, if an approximate calculation from the last relationship led to an approximate solution for a linear ordinary differential system, our interval analysis provides more rigorous framework. Then, the strict enclosure of the solution would be obtained by interval computation of the matrix enclosing the exponentiation of the matrix of interval $A$. The most obvious way to get an interval enclosure of $\exp (A)$ is to evaluate the truncated Taylor series using interval computation and to bind the rest. But the problem is that the results obtained by the exponential of Taylor and the exponential Horner with intervals computation often diverge especially the truncated Taylor series. For this reason, three implementations of exponentials (Exponential of Taylor, Exponential of Horner, and Exponential of Horner with reduction) given in $[40,41]$ are tested in our study. Its use in conjunction with interval analysis that allows us to control rounding errors to properly criticize and evaluate the results obtained by each exponential in the example of MacPherson suspension system will discussed in Sect. 6.1.2. The evaluation of those three implementations of exponential was made by programming in Matlab with the intervals library IntLab [42].

\subsection{Taylor Series}

The Taylor series is applicable only when the matrix has fixed values (like any diagonalizable matrix in $\mathbb{C}$ and the exponential of a complex is known. This method is very easy to implement). Unfortunately when it comes apart, this definition is no longer valid (we can not define an interval of eigenvalue due to its discontinuous nature). The easiest way is to apply the Taylor series:

$\exp (A)=\sum_{k=0}^{\infty} \frac{A^{k}}{k !}$

Let's give an example to evaluate its accuracy [30] and show that the truncation of the Taylor series is not well suited to the interval evaluation, even if no truncation of the series is made. We compute the formal expression of the exponential of the matrix $A(t)$ for all $t \in[-9 ;-7]$ with the eigenvalue method for its upper and lower limits. We find a misleading result because the lower and upper bounds can't be improved. This means $[\exp (A), \overline{\exp (A)}]$ is the best enclosure of $\exp (A)$.

$A=\left[\begin{array}{ll}t & 0 \\ 1 & 2\end{array}\right] \quad \exp (A)=\left[\begin{array}{cc}0.0001234 ; 0.0009119] & 1 \\ 2.7183 & 7.3891\end{array}\right]$

Now we make the calculation with the Taylor series truncated to 18 terms with a rest practically negligible that can be estimated less than:

$\rho(\|A\|, n) \times[-9,-7]=\frac{\mid A \|^{n+1}}{(n+1) ! \times\left(1-\frac{\mid A \|}{n+2}\right)} \times[-9 ;-7]$ 
We get disappointing results as we can see below:

$\exp (A)=\left[\begin{array}{cc}{[-5103.560 ; 5105.246]} & 0 \\ {[-486.589 ; 487.898]} & 7.389\end{array}\right]$

As shown in the example above, even with a fairly high order for the expansion of the matrix [A], the influence of the rest is insignificant. So the evaluation of the Taylor exponential shows that it enlarges the limits of a matrix in an intervals calculation. The reason for this bad behavior of Taylor series in assessment by interval is the loss of dependency between the different expression occurrences of variables that occur during each assessment interval. Two well-known techniques can help to reduce the pessimism in the evaluation of the Taylor series by interval. First, a matrix whose intervals are centered forms which can provide results especially for very small input intervals. Such centered form for the exponential of a matrix which wass proposed in [43-45]. However, this centered assessment dedicated to exponentiation of interval matrix is very complex and difficult to follow or implement. The second technique is to rewrite the formal expression in order to obtain a more proportionate approach to the interval evaluation (usually by reducing the number of occurrences of variables). For example, the evaluation of a polynomial in Horner form is known to improve the assessment interval [35]. It can be naturally applied to the Taylor series in the exponential of a matrix, and was already used [44] to calculate the exponential of a matrix center as requested in the centered form. Proof of the best adaptation of the Horner exponential is provided in the following paragraph. We can conclude then that Taylor exponential must be used when there are a certain homogeneity in terms of the matrix and that its standard is not very important (the number of terms in the series must be chosen from the relation $n+2>\|A\|)$. Its bad behavior is attributed to a high number of operations in intervals evaluation.

\subsection{Horner exponential}

To reduce the number of operations, Goldsztejn [40,42] applied the polynomial factorization of Horner [40] in Taylor series. This method consists in the evaluation of the polynomial image, at a point of the Euclidean space and performing several divisions. Specifically, instead of having a sum with power to assess, a product of simple terms that will reduce the number of occurrences of the terms of the matrix, is obtained. So the computation time is reduced and the cost of the computing operations is minimized. The exponential scheme of Horner is the following:

$$
\exp (A)=I+A \cdot\left(I+\frac{A}{2}\left(I+\frac{A}{3}\left(\ldots\left(I+\frac{A}{n}\right)\right)\right)\right)
$$

However the rest is the same as that of the Taylor exponential (this was expected given that the writing comes from the expression of Taylor series). If we take the example of the previous section, we find for $n=18$ (with a rest below $10^{-3}$ ):

$\exp (A)=\left[\begin{array}{cc}{[-474.602 ; 476.288]} & 0 \\ {[-67.172 ; 68.481]} & 7.389\end{array}\right]$

We have now a better framework as we move the component 2.1 from [486.589; 487.898] to [-67.172; 68.481]. This is an improvement in the calculation of the exponential. In addition, it is shown that the loss of dependency due to the significant improvement of the evaluation range, offering more accuracy and less expensive calculations. As explained previously, the center evaluation (centered matrix) provides more distinct enclosures but for the resolution of our problem is not yet clear. Therefore we turn to another digital trick.

\subsection{Horner exponentially with reduction}

The objective here is to reduce the intervals to get a better precision in the calculations. The digital trick [40] consists in writing the exponential as follows:

$\exp (A)=\left(\frac{A}{2^{s}}\right)^{2^{s}}$

When doing so, both the norm of the matrix and the intervals are reduced to the value we want. The number of terms to be chosen is determined by the following equation $(n+2) .2 s .\|A\|$ and can therefore be reduced depending on the value, without significant degradation of the results. But we have to be careful, when we choose a too big $s$ because in the calculation of the power, the computer can interpret this value as infinite, which irretrievably distorts the result. If we take the example of the matrix A we obtain for $n=18$ and $s=40$ a very satisfying results as we can see below:

$\exp (A)=\left[\begin{array}{cc}{[-0.000241 ; 0.000912]} & 0 \\ {[0.656588 ; 0.821840]} & 7.389\end{array}\right]$

We get for the same number of terms and a reduced number of operations, better results than the two previous exponentials. It therefore seems a priori that the Horner exponential with reduction is a suitable method to solve a linear differential equation. But to date, this approach, developed by Goldsztejn in [40], has been tested only on small matrix $($ size $=2,2)$ as previously shown. One of our goals is to assess it on a real dynamic system. However, to confirm these results, we will test all the previous types of exponentials to simulate the behavior of a MacPherson suspension system. 


\section{MacPherson suspension system}

Suspension systems cover several areas of applications. This involves different operating environments, ranging from domestic uses (office chairs ...) to applications with high reliability and requirement (vehicle). Depending on the application for which is dedicated to the suspension system, one or more objectives of pre-sizing are often specified and must be optimized.

The roles of a suspension system are to support the weight of the vehicle to isolate the vibrations from the road and to maintain the traction between the tire and the road.

In today's automotive industry, the optimization $[49,50]$ of a vehicle in terms of comfort and security plays an active role. This involves performing a large number of simulations to find more efficient and innovative solutions, taking into account the time constraint. Yet, despite the diversity of existing tools and methods, the simulation step in most complex systems is relatively long. One of the main purposes of this study was to apply the interval computation method on a MacPherson suspension system Fig. 4 to determine several solutions, which offer the possibility to choose the ideal settings from existing solutions which satisfies all the imposed constraints.

\subsection{Dynamic model of the arm of Macpherson suspension system}

This study is based on the dynamic model of Sohn et al. [40] for MacPherson suspension system shown in Fig. 4. It is composed of a quarter vehicle body, an axle and a tire, a coil spring, a damper, an axle, a load disturbance and a control arm. The quarter vehicle body is assumed to have only a vertical motion. If the mass of the control arm is ignored and the joint between the control arm and the car body is

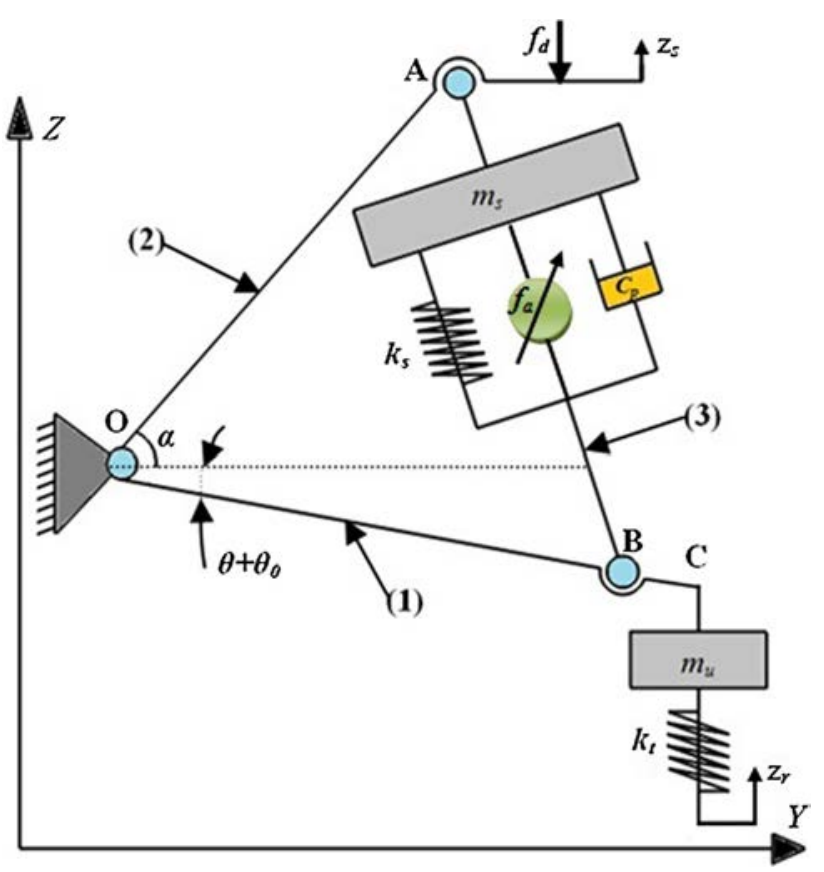

Fig. 5 Dynamic quarter vehicle model of a Macpherson suspension

assumed to be a pin joint, the degrees of freedom is two. As the mass of the control arm is much smaller than those of the sprung mass and the unsprung mass, it can be neglected. Under the above assumption, a model of the Macpherson type suspension system is introduced in Fig. 5. The vertical displacement $z_{s}$ of the sprung mass and the rotation angle $\theta$ of the control arm are chosen as the generalized coordinates. The assumptions adopted in this modelling are summarized as follows: The sprung mass has only the vertical displacement while horizontal movement is ignored $z_{s}$. The unsprung mass (spindle and tire) is connected to the vehicle body through the damper and spring as well as the control arm in two ways. $\theta$ denotes the angular displacement of the
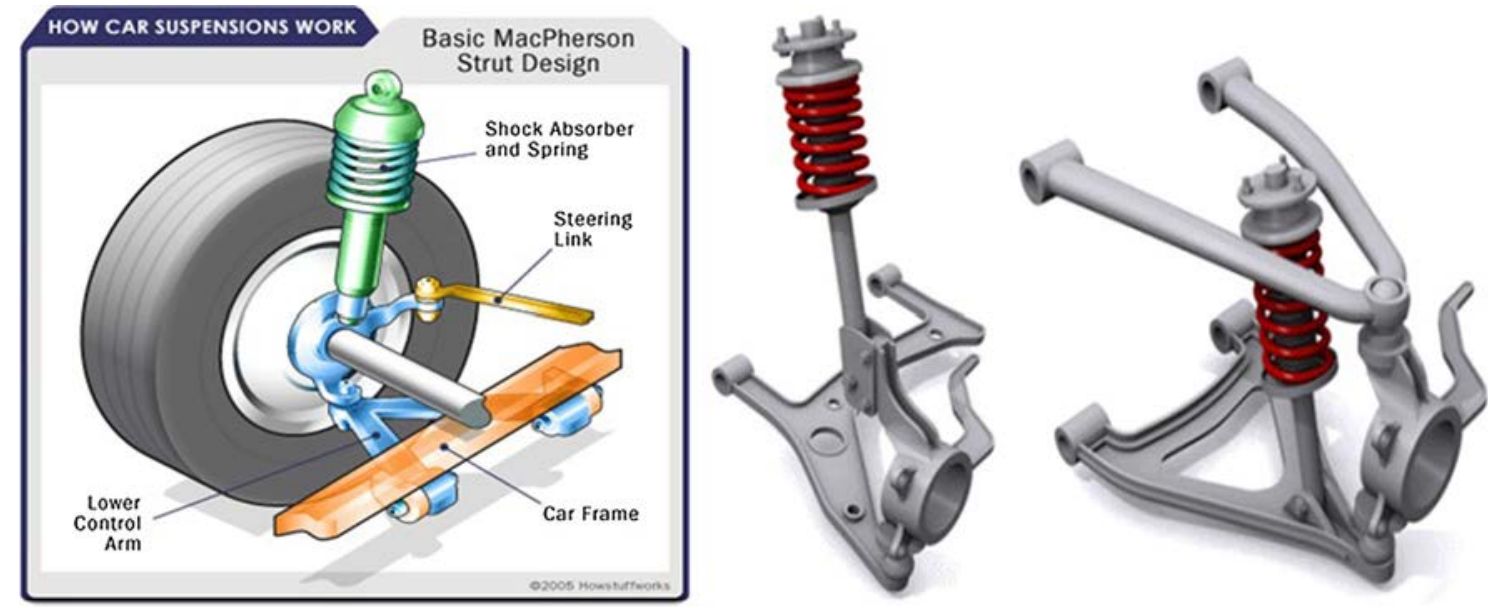

Fig. 4 Macpherson suspension system [47,48] 
control arm. The values of $z_{s}$ and $\theta$ will be measured from their static equilibrium points.The sprung and the unsprung masses are assumed to be particles. The mass and the stiffness of the control arm are neglected. The coil spring deflection, the tire deflection and the damping forces are in the linear regions of their operating ranges.

Considering $\left(y_{A}, Z_{A}\right),\left(y_{B}, Z_{B}\right)$ and $\left(y_{C}, Z_{C}\right)$ denote the coordinates of point $\mathrm{A}, \mathrm{B}$ and $\mathrm{C}$, respectively, when the suspension system is at an equilibrium point. Considering the sprung mass be translated upward by $z_{s}$, and the unsprung mass be rotated by $\theta$ in the counter-clockwise direction. Then, the following equations hold.

$$
\begin{aligned}
& y_{A}=0 \\
& z_{A}=z_{s} \\
& y_{B}=l_{B}\left(\cos \left(\theta-\theta_{0}\right)-\cos \left(-\theta_{0}\right)\right) \\
& z_{B}=z_{s}+l_{B}\left(\sin \left(\theta-\theta_{0}\right)-\sin \left(-\theta_{0}\right)\right) \\
& y_{C}=l_{C}\left(\cos \left(\theta-\theta_{0}\right)-\cos \left(-\theta_{0}\right)\right) \\
& z_{C}=z_{s}+l_{C}\left(\sin \left(\theta-\theta_{0}\right)-\sin \left(-\theta_{0}\right)\right)
\end{aligned}
$$

Where $\theta_{0}$ is the initial angular displacement of the control arm at an equilibrium point. Considering $\alpha^{\prime}=\alpha+\theta_{0}$. Then, the following relations are obtained from the triangle $\mathrm{OAB}$.

$$
\begin{aligned}
& l=\left(l_{A}^{2}+l_{B}^{2}+2 l_{A} l_{B} \cos \alpha^{\prime}\right)^{\frac{1}{2}} \\
& l^{\prime}=\left(l_{A}^{2}+l_{B}^{2}+2 l_{A} l_{B} \cos \left(\alpha^{\prime}-\theta\right)\right)^{\frac{1}{2}}
\end{aligned}
$$

Where $l$ is the initial distance from A to B at an equilibrium state, and $l^{\prime}$ is the changed distance with the rotation of the control arm by $\theta$. Therefore, the deflection of the spring, the relative velocity of the damper and the deflection of the tire are, respectively

$$
\begin{aligned}
(\Delta l)^{2}= & \left(l-l^{\prime}\right)^{2} \\
= & 2 a_{1}-b_{1}\left(\cos \alpha^{\prime}+\cos \left(\alpha^{\prime}-\theta\right)\right)-2\left[a_{l}^{2}-a_{l} b_{l} .\right. \\
& \left.\quad\left(\cos \alpha^{\prime}+\cos \left(\alpha^{\prime}-\theta\right)+b_{l}^{2} \cos \alpha^{\prime} \cos \left(\alpha^{\prime}-\theta\right)\right)\right]
\end{aligned}
$$

$$
\begin{aligned}
& (\Delta l)^{\cdot}=\left(l^{\cdot}-l^{\prime}\right)=\frac{b_{l} \sin \left(\alpha^{\prime}-\theta\right) \theta^{\cdot}}{2\left(a_{l}-b_{l} \cos \left(\alpha^{\prime}-\theta\right)\right)^{\frac{1}{2}}} \\
& z_{C}-z_{r}=z_{s}+l_{C}\left(\sin \left(\theta-\theta_{0}\right)-z_{r}\right.
\end{aligned}
$$

Where, $a_{l}=l_{A}^{2}+l_{B}^{2}, b_{l}=2 l_{A} l_{B}$

\subsubsection{Equations of motion}

Lagrange's method is used to obtain the equations of motion. Considering $T, V$ and $D$ denote the kinetic energy, the potential energy and the damping energy of the system, respectively. Then, these are

$$
\begin{aligned}
T & =\frac{1}{2} m_{s} z_{s}^{2}+\frac{1}{2} m_{u}\left(y_{C}^{{ }^{2}}+Z_{C}^{{ }^{2}}\right) \\
V & =\frac{1}{2} k_{s} \Delta l^{2}+\frac{1}{2} k_{t}\left(z_{C}+z_{r}\right)^{2} \\
D & =\frac{1}{2} c_{p}\left(\Delta l^{\cdot}\right)^{2}
\end{aligned}
$$

Substituting the derivatives of $(9),(10)$ and $(13,14,15)$ into $(16,17,18)$ yields

$$
\begin{aligned}
& T=\frac{1}{2}\left(m_{s}+m_{u}\right) Z_{s}^{2}+\frac{1}{2} m_{u} l_{C}^{2} \theta^{\cdot 2}+m_{u} l_{C} \cos \theta \theta^{\cdot} z_{s}^{*} \\
& V=\frac{1}{2} k_{s}\left[2 a_{l}-b_{l}\left(\cos \alpha^{\prime}+\cos \left(\alpha^{\prime}-\theta\right)\right)-2\left(a_{l}^{2}-a_{l} b_{l} .\right.\right. \\
& \left.\left.\cos \alpha^{\prime}+\cos \left(\alpha^{\prime}-\theta\right)+b_{l}^{2} \cos \left(\alpha^{\prime}-\theta\right)\right)^{\frac{1}{2}}\right] . \\
& \frac{1}{2} k_{t}\left[z_{s}+l_{C}\left(\sin \left(\theta-\theta_{0}\right)-\sin \left(-\theta_{0}\right)\right)-z_{r}\right]^{2} \\
& D=\frac{c_{p} b_{l}^{2} \sin ^{2}\left(\alpha^{\prime}-\theta\right) \theta^{\cdot}}{8\left(a_{l}-b_{l} \cos \left(\alpha^{\prime}-\theta\right)\right)}
\end{aligned}
$$

Finally, for the two generalized coordinates $q_{1}=z_{s}$ and $q_{2}=\theta$, the equations of motion are obtained as follows:

$$
\begin{aligned}
& \left(m_{s}+m_{u}\right) z_{s}^{\cdot}+m_{u} l_{C} \cos \left(\theta-\theta_{0}\right) \theta^{\cdot} \\
& \quad-m_{u} l_{C} \sin \left(\theta-\theta_{0}\right) \theta^{\cdot 2}+k_{t}\left[z_{s}-z_{r}\right. \\
& \left.\quad+l_{C}\left(\sin \left(\theta-\theta_{0}\right)-\sin \left(-\theta_{0}\right)\right)\right]=-f_{d} \\
& m_{u} l_{C}^{2} \theta^{\cdot}+m_{u} l_{C} \cos \left(\theta-\theta_{0}\right) z_{s}^{\cdot}+\frac{c_{p} b_{l}^{2} \sin ^{2}\left(\alpha^{\prime}-\theta_{0}\right) \theta^{\cdot}}{4\left(a_{l}-b_{l} \cos \left(\alpha^{\prime}-\theta\right)\right)} \\
& \quad-k_{t} l_{C} \cos \left(\theta-\theta_{0}\right)\left(z_{s}+l_{C}\left(\sin \left(\theta-\theta_{0}\right)-\sin \left(-\theta_{0}\right)\right)\right. \\
& \left.\quad-z_{r}\right)-\frac{1}{2} k_{s} \cos \left(\alpha^{\prime}-\theta\right)\left[b_{l}+\frac{d_{l}}{\left(c_{l}-d_{l} \cos \left(\alpha^{\prime}-\theta\right)^{\frac{1}{2}}\right)}\right] \\
& =-l_{B} f_{a}
\end{aligned}
$$

Where,

$c_{l}=a_{l}^{2}-a_{l} b_{l} \cos \left(\alpha+\theta_{0}\right)$ and $d_{l}=a_{l} b_{l}-b_{l}^{2} \cos \left(\alpha+\theta_{0}\right)$

Now, introducing the state variables as $\left[\begin{array}{llll}x_{1} & x_{2} & x_{3} & x_{4}\end{array}\right]=\left[\begin{array}{ll}z_{s} \\ y_{s}\end{array}\right.$ $\left.z_{s}^{*} \theta \theta^{*}\right]$. Then, 22-23 can be written in the state equation as follows:

$x_{1}=x_{2}$

$x_{2}^{*}=f_{1}\left(x_{1}, x_{2}, x_{3}, x_{4}, f_{a}, z_{r}, f_{d}\right)$

$x_{3}^{*}=x_{4}$

$x_{4}^{*}=f_{2}\left(x_{1}, x_{2}, x_{3}, x_{4}, f_{a}, z_{r}, f_{d}\right)$

where,

$$
\begin{aligned}
f_{1}= & \frac{1}{D_{1}}\left[m_{u} l_{C}^{2} \sin \left(x_{3}-\theta_{0}\right) x_{4}^{2}+\frac{1}{2} k_{s} \sin \left(\alpha^{\prime}-x_{3}\right) .\right. \\
& \cos \left(x_{3}-\theta_{0}\right) g\left(x_{3}\right)+c_{p} h\left(x_{3}\right) \theta^{\cdot}-k_{t} l_{C} \sin ^{2}\left(x_{3}-\theta_{0}\right) . \\
& \left.z(.)+l_{B} f_{a} \cos \left(x_{3}-\theta_{0}\right)-l_{C} f_{d}\right]
\end{aligned}
$$




$$
\begin{aligned}
f_{2}= & \frac{1}{D_{2}}\left[m_{u}^{2} l_{C}^{2} \sin \left(x_{3}-\theta_{0}\right) \cos \left(x_{3}-\theta_{0}\right) x_{4}^{2}+\left(m_{s}+m_{u}\right) .\right. \\
& c_{p} h\left(x_{3}\right) x_{4}-\frac{1}{2}\left(m_{s}+m_{u}\right) k_{s} \sin \left(\alpha^{\prime}-x_{3}\right) g\left(x_{3}\right)+m_{s} k_{t} . \\
& l_{C} \cos \left(x_{3}-\theta_{0}\right) z(.)+\left(m_{s}+m_{u}\right) l_{B} f_{a} \\
& \left.-m_{u} l_{C} \cos \left(x_{3}-\theta_{0}\right) f_{d}\right]
\end{aligned}
$$

\section{And}

$$
\begin{aligned}
D_{1} & =m_{s} l_{C}+m_{u} l_{C} \sin ^{2}\left(x_{3}-\theta_{0}\right) \\
D_{2}= & m_{s} m_{u} l_{C}^{2}+m_{u}^{2} l_{C}^{2} \sin ^{2}\left(x_{3}-\theta_{0}\right) \\
g\left(x_{3}\right)= & b_{l}+\frac{d_{l}}{\left(c_{l}-d_{l} \cos \left(\alpha^{\prime}-x_{3}\right)^{\frac{1}{2}}\right)} \\
h\left(x_{3}\right)= & \frac{b_{l}^{2} \sin ^{2}\left(\alpha^{\prime}-x_{3}\right)}{4\left(a_{l}-b_{l} \cos \left(\alpha^{\prime}-x_{3}\right)\right)} \\
z(.)= & z\left(x_{1}, x_{2}, z_{r}\right)=x_{1} \\
& +l_{c}\left[\sin \left(x_{3}-\theta_{0}\right)-\sin \left(-\theta_{0}\right)\right]-z_{r}
\end{aligned}
$$

\subsubsection{Linearization}

In order to simplify of the resolution the equations of the system, (20) is linearized at the equilibrium state where $x_{e}=\left(x_{1 e}, x_{2 e}, x_{3 e}, x_{1 e}\right)=(0,0,0,0)$. Then, the resulting linear equation is

$x^{\bullet}=A x(t)+B_{1} f_{a}(t)+B_{2} z_{r}(t)+B_{3} f_{d}(t), x(0)=x_{0}$

where,

$$
\begin{aligned}
& A=\left[\begin{array}{cccc}
0 & 1 & 0 & 0 \\
\frac{\partial f_{1}}{\partial x_{1}} & \frac{\partial f_{1}}{\partial x_{2}} & \frac{\partial f_{1}}{\partial x_{3}} & \frac{\partial f_{1}}{\partial x_{4}} \\
0 & 0 & 0 & 1 \\
\frac{\partial f_{2}}{\partial x_{1}} & \frac{\partial f_{2}}{\partial x_{2}} & \frac{\partial f_{2}}{\partial x_{3}} & \frac{\partial f_{2}}{\partial x_{4}}
\end{array}\right]=\left[\begin{array}{cccc}
0 & 1 & 0 & 0 \\
a_{21} & 0 & a_{23} & a_{24} \\
0 & 0 & 0 & 1 \\
a_{41} & 0 & a_{43} & a_{44}
\end{array}\right] \\
& B_{1}=\left[\begin{array}{c}
0 \\
\frac{\partial f_{1}}{\partial f_{a}} \\
0 \\
\frac{\partial f_{2}}{\partial f_{a}}
\end{array}\right]=\left[\begin{array}{c}
\frac{l_{B} \cos \left(-\theta_{0}\right)}{m_{s} l_{C}+m_{u} l_{C} \sin ^{2}\left(-\theta_{0}\right)} \\
0 \\
\frac{\left(m_{s}+m_{u}\right) l_{B}}{m_{s} m_{u} l_{C}^{2}+m_{u}^{2} l_{C}^{2} \sin ^{2}\left(-\theta_{0}\right)}
\end{array}\right] \\
& B_{2}=\left[\begin{array}{c}
0 \\
\frac{\partial f_{1}}{\partial z_{r}} \\
0 \\
\frac{\partial f_{2}}{\partial z_{r}}
\end{array}\right]=\left[\begin{array}{c}
\frac{k_{t} l_{C} \sin ^{2}\left(-\theta_{0}\right)}{m_{s} l_{C}+m_{u} l_{C} \sin ^{2}\left(-\theta_{0}\right)} \\
0 \\
\frac{m_{s} k_{t} l_{C} \cos \left(-\theta_{0}\right)}{m_{s} m_{u} l_{C}^{2}+m_{u}^{2} l_{C}^{2} \sin ^{2}\left(-\theta_{0}\right)}
\end{array}\right]
\end{aligned}
$$

$$
B_{3}=\left[\begin{array}{c}
0 \\
\frac{\partial f_{1}}{\partial f_{d}} \\
0 \\
\frac{\partial f_{2}}{\partial f_{d}}
\end{array}\right]=\left[\begin{array}{c}
0 \\
\frac{l_{C}}{m_{s} l_{C}+m_{u} l_{C} \sin ^{2}\left(-\theta_{0}\right)} \\
0 \\
\frac{m_{u} l_{C} \cos \left(-\theta_{0}\right)}{m_{s} m_{u} l_{C}^{2}+m_{u}^{2} l_{C}^{2} \sin ^{2}\left(-\theta_{0}\right)}
\end{array}\right]
$$

with

$$
\begin{aligned}
a_{21}= & \frac{-k_{t} l_{C} \sin ^{2}\left(-\theta_{0}\right)}{D_{1}} \\
a_{23}= & \frac{1}{D_{1}^{2}}\left[\left[\frac{1}{2} k_{s}\left(b_{l}+\frac{d_{l}}{\left(c_{l}-d_{l} \cos \alpha^{\prime}\right)^{\frac{1}{2}}}\right) \cos \left(\alpha^{\prime}+\theta_{0}\right)\right.\right. \\
& -\frac{1}{2}\left(k_{s} \sin \alpha^{\prime} \cos \left(-\theta_{0}\right)\right) \cdot\left(\frac{d_{l}^{2} \sin \alpha^{\prime}}{2\left(c_{l}-d_{l} \cos \alpha^{\prime}\right)^{\frac{3}{2}}}\right) \\
& \left.-k_{t} l_{C}^{2} \sin ^{2}\left(-\theta_{0}\right) \cos \left(-\theta_{0}\right)\right] \cdot\left[m_{s} l_{C}+m_{u} l_{C} \sin ^{2}\left(-\theta_{0}\right)\right] \\
& \left.+m_{u} k_{s} l_{C} \sin ^{\prime} \sin \left(-\theta_{0}\right) \cos ^{2}\left(-\theta_{0}\right)\left(b_{l}+\frac{d_{l}}{\left(c_{l}-d_{l} \cos \alpha^{\prime}\right)^{\frac{1}{2}}}\right)\right] \\
a_{24}= & \frac{1}{D_{1}} \cdot \frac{c_{p} b_{l}^{2} \sin ^{2} \alpha^{\prime}}{4\left(a_{l}-b_{l} \cos \alpha^{\prime}\right)}
\end{aligned}
$$

$$
\begin{aligned}
a_{41}= & -\frac{m_{s} k_{t} l_{C} \cos \left(-\theta_{0}\right)}{D_{2}} \\
a_{43}= & \frac{1}{D_{1}^{2}}\left[\left[\frac{1}{2}\left(m_{s}+m_{u}\right) k_{s} \cos \alpha^{\prime}\left(b_{l}+\frac{d_{l}}{\left(c_{l}-d_{l} \cos \alpha^{\prime}\right)^{\frac{1}{2}}}\right)\right.\right. \\
& -\frac{1}{2}\left(m_{s}+m_{u}\right) k_{s} \sin \alpha^{\prime} \cdot\left(\frac{d_{l}^{2} \sin \alpha^{\prime}}{2\left(c_{l}-d_{l} \cos \alpha^{\prime}\right)^{\frac{3}{2}}}\right) \\
& \left.-m_{s} k_{t} l_{C}^{2} \cos \left(-\theta_{0}\right)\right] \cdot\left[m_{s} m_{u} l_{C}^{2}+m_{u}^{2} l_{C}^{2} \sin ^{2}\left(-\theta_{0}\right)\right] \\
& \left.+\frac{1}{2}\left(m_{s}+m_{u}\right) m_{u}^{2} k_{s} l_{C}^{2} \sin \alpha^{\prime} \sin \left(-\theta_{0}\right)\left(b_{l}+\frac{d_{l}}{\left(c_{l}-d_{l} \cos \alpha^{\prime}\right)^{\frac{1}{2}}}\right)\right]
\end{aligned}
$$$$
a_{44}=-\frac{1}{D_{2}} \cdot \frac{\left(m_{s}+m_{u}\right) c_{p} b_{l}^{2} \sin ^{2} \alpha^{\prime}}{4\left(a_{l}-b_{l} \cos \alpha^{\prime}\right)}
$$

\section{Dynamic simulation of a passive Macpherson suspension system}

\subsection{Transient state}

\subsubsection{Fixed values}

We handle the case where we impose a displacement of $10 \mathrm{~cm}$ on the wheel (the differential equations are with no second term), so the system is written as following:

$$
x^{*}=A \cdot x(t) \quad \text { With }: x^{*}=\left[\begin{array}{c}
z_{s} \\
z_{s}^{*} \\
\theta \\
\theta \cdot
\end{array}\right]
$$


Table 1 Design parameters of the MacPherson suspension system

\begin{tabular}{lll}
\hline$m_{u}$ & Wheel mass & $25 \mathrm{~kg}$ \\
$m_{s}$ & Vehicle mass & $500 \mathrm{~kg}$ \\
$k_{t}$ & Tire stiffness & $200000 \mathrm{~N} / \mathrm{m}$ \\
$k_{s}$ & Damper stiffness & $20000 \mathrm{~N} / \mathrm{m}$ \\
$c_{p}$ & Viscous damping coefficient & $2000 \mathrm{~N} . \mathrm{s} / \mathrm{m}$ \\
\hline
\end{tabular}
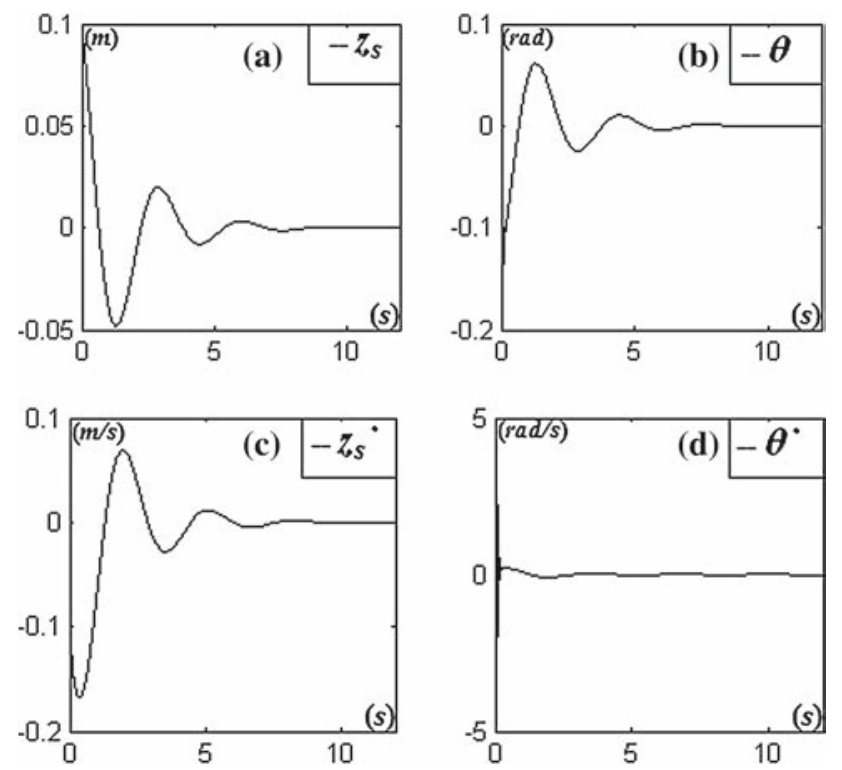

Fig. 6 MacPherson suspension system response when excited with snap-displacement. a Vertical displacement of mass $m_{s}$. b Rotation of mass $m_{s}$ around $X$. c Linear velocity of mass $m_{s}$. d Angular velocity of $\operatorname{mass} m_{s}$

The system is shaped to apply the exponential matrix. Thus, without introducing uncertainty in the values of MacPherson suspension parameters (the system is solved with the method of exponential eigenvalues).

The numerical values of the design variables of the MacPherson suspension system used for the simulation of its dynamic behavior are represented in Table 1 .We obtain the reference curves shown in Fig. 6.

The dynamic behavior of a Macpherson suspension system when excited by a displacement of $10 \mathrm{~cm}$ on the wheel is represented in Fig. 6. The displacement $z_{s}$ converge to zero, which is normal as the role of a suspension is to mitigate the vibrations caused by road in a minimum time. We notice that the chassis takes $8 \mathrm{~s}$ to stabilize and to attain its equilibrium position, which is not a long period considering the magnitude of excitation and the fact that the type of MacPherson suspension system is a passive suspension in this case. This is due to the fact that the design parameters are well chosen specifically on the chassis stiffness $k_{s}$ which quickly stiffens the structure. Also we notice the variation of the rotation angle $\theta$ is very small, it takes $8 \mathrm{~s}$ to attain its equilibrium position like the vertical displacement $z_{s}$. The variation of the linear and angular velocity $\left(z_{s}^{*}, \theta^{*}\right)$ is very small compared to its equilibrium position, which implies that the damping coefficient $c_{p}$ is well chosen. Although the dynamic behavior of the MacPherson suspension responded well for the prospects expected but we are not sure if the design parameters chosen are the best. So in next paragraph we introduced uncertainty in these parameters.

\subsubsection{Uncertainties}

In this sub-section the evaluation of the dynamic behavior of the MacPherson suspension system was studied with uncertainty. The uncertainties have been applied to the values of design parameters which define the dynamic model of the MacPherson suspension system. The system of differential equations is solved with the interval Based Simulation method. The same calculation used in Sect. 6.1.1 is repeated but with uncertainty in reference values to determine the behavior of the suspension system by interval. Typically, for a first test, a $0.2 \%$ uncertainty on the stiffness $k_{s}$ and $0.4 \%$ on the stiffness $k_{t}, 0.3 \%$ uncertainty on the mass $m_{s}$ and $0.1 \%$ on the mass $m_{u}$ and $0.5 \%$ on the damping coefficient $c_{p}$ were imposed. The areas of these design parameters are as follows:

$k_{t}=[199200 ; 200800] m_{u}=[24.975 ; 25.025]$

$c_{p}=[1990 ; 2010] k_{s}=[19960 ; 20040] m_{s}=[495.5 ; 501.5]$

The calculation has been done in MATLAB with the intervals library IntLab [38] for a number of terms equal to 14 (with Taylor, Horner or Horner with reduction) [20] and a reduction factor equal to 45 . The results are shown in Fig. 7.

In Fig. 7 the behavior of the Macpherson suspension system when excited by an instantaneous displacement is represented. With the Interval Based Simulation we determine an envelope which includes a set of performance for displacement $z_{s}$ and rotational movement $\theta$ of chassis 1 . For the calculations of the dynamic response of the MacPherson suspension with uncertainty, two types of exponential have been applied. The calculations that have been made with Taylor (Fig. 7a, b) and Horner exponential (Fig. 7c, d).

We find that the results shown in the previous figures do not correspond to reality, since a significant difference (about $10^{30}$ ) identified between the curves calculated with setting values and curves calculated with uncertainty. So as mentioned in the Sect. 4, the calculation by interval with the use of Taylor exponential or and Horner exponential diverges which confirms the divergence shape of curves of displacements $z_{s}$ and rotational movement $\theta$. However, we notice that curves obtained by Taylor deviates in a increasing manner in a single direction while curves calculated by the Horner exponential deviates also in two directions in a symmetrical manner which is close to reality since the references curves 

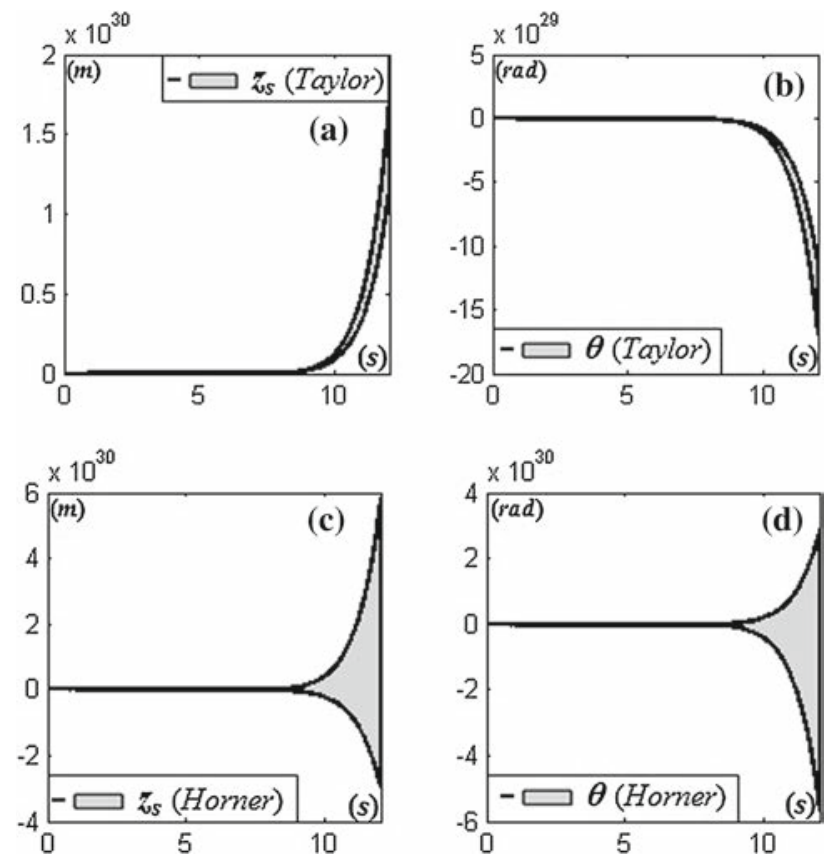

Fig. 7 The behavior of Macpherson suspension system when excited by an instantaneous displacement (comparison between the response calculated with the use of Taylor exponential and Horner exponential). a Vertical displacement of mass $m_{s}$ calculated by applying Taylor exponential. b Rotation of mass $m_{s}$ around $X$ calculated by applying Taylor exponential. c Vertical displacement of mass $m_{s}$ calculated by applying Horner exponential. d Rotation of mass $m_{s}$ around $X$ calculated by applying Horner exponential

have a sinusoidal pseudo-periodic form, thus confirming the theory outlined in Sect. 4.

In Fig. 8 the Horner exponential with reduction is tested to calculate the dynamic behaviour of MacPherson suspension. We notice that the set of solutions obtained are converging and have the same appearance like the curve calculated with fixed values which confirms the theories in Sect. 4 [40]. To ascertain the validity of the algorithm, the uncertainties were set to zero to check that the same curves were well received. However, the results obtained with the use of the mathematical formulation of Horner exponentially with reduction reflect that when the range of variables is divided into multiple sections, it will significantly reduce the error propagation. So we can conclude that the expression of Horner exponential with reduction is the most adaptable to the mathematical formulation to make exponential calculation by intervals. This asserts that the divergence of results is not due to the Interval based Simulation method but to the mathematical formulation of the problem.

\subsection{Harmonic state}

\subsubsection{Setting values}

Just like what has been achieved previously in Sect. 6.1.1, the same process and calculations are repeated in the next,
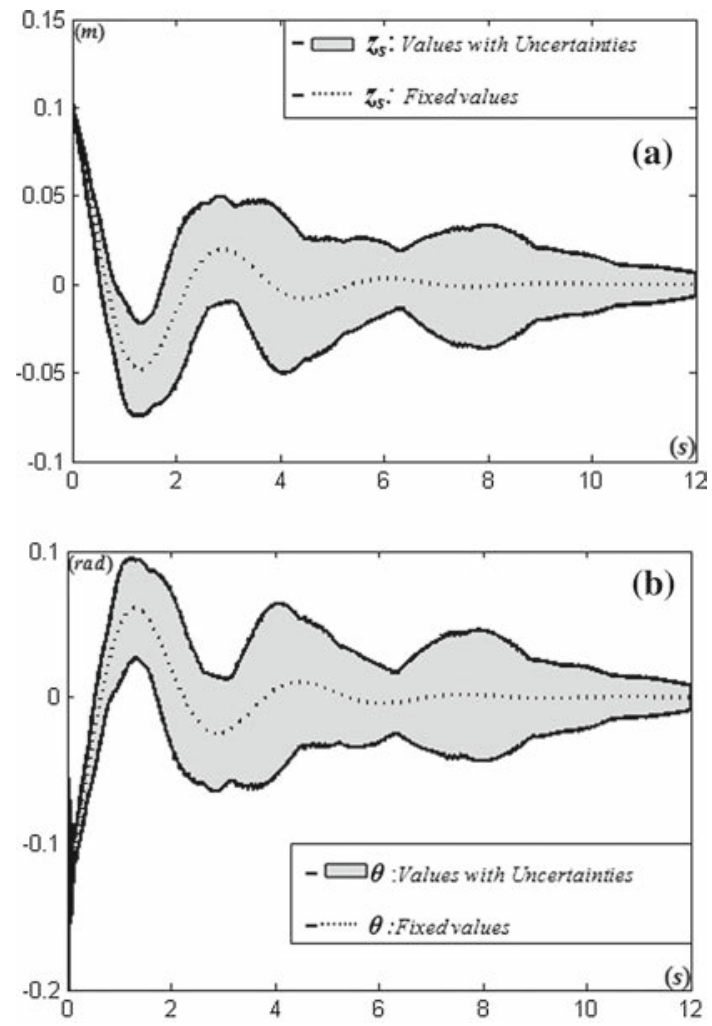

Fig. 8 The behavior of Macpherson suspension system when excited by an instantaneous displacement (response calculated with the use of exponential Horner with reduction). a Vertical displacement of mass $m_{s}$ calculated by applying Horner exponential with reduction. b Rotational movement of mass $m_{s}$ calculated by applying Horner exponential with reduction

except for the excitation of the dynamic model which has been done besides the displacement of $10 \mathrm{~cm}$ imposed on the wheel with a sinusoidal force. Repeating the previous matrices, the system to solve is:

$x^{\cdot}=A x(t)+B_{1} f_{a}(t)+B_{2} \frac{F(t)}{k_{t}}+B_{3} f_{d}(t)$

With:

$F(t)=0.02 \cdot k_{t} \cdot \sin (10 t)$ Even with zero initial conditions.

And:

$f_{a}=f_{d}=0$

The solution of this system is the following form

$x(t)=e^{A \cdot t} \cdot x_{0}+\int_{0}^{t} e^{A \cdot \lambda} \cdot B_{2} \cdot \frac{F(t-\lambda) .}{k_{t}} \cdot d \lambda$

After an integration by parts we find the following solution:

$x(t)=e^{A . t} \cdot x_{0}+\frac{B_{2} \cdot K}{\omega\left[1+\left(\frac{A}{\omega}\right)^{2}\right]}\left(e^{A . t}-\cos \omega \cdot t-\frac{A}{\omega} \sin \omega . t\right)$ 

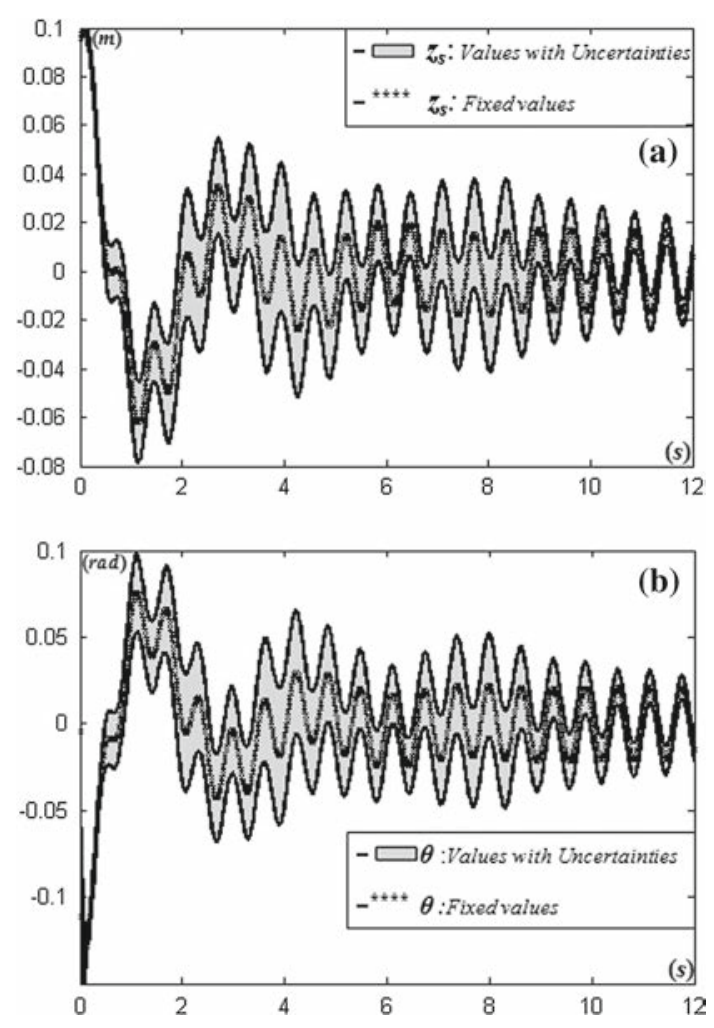

Fig. 9 The behavior of Macpherson suspension system when excited by an instantaneous displacement (response calculated with the use of exponential Horner with reduction). a Vertical displacement of mass $m_{s}$ calculated by applying Horner exponential with reduction. b Rotational movement of mass $m_{s}$ calculated by applying Horner exponential with reduction

The Behavior of a Macpherson suspension system when excited by a sinusoidal force is represented in Fig. 9. The stars point curves $\left(-* * *_{-}\right)$represent the calculations which have been done with fixed values. So according to these results in Fig. 8 we notice that the curves $z_{s}$ and $\theta$ have the expected behaviours. They are sinusoids curves that follow the road as they have the same frequency and nearly the same amplitude. In addition, there is an angle shift between $z_{s}$ and the sinusoidal force of excitation $F$. It caused by the difference time between the instant when the peak occurred and the instant when users have felt it. Fortunately this is a part of the role of the suspension to delay and reduce the abrupt changes of the road. We can therefore use these new curves as reference curves.

\subsubsection{Uncertainties}

Uncertainties defined in Sect. 6.1.2 are now included but with the same percentages. According to results in Sect. 6.2.1. We apply just the Horner exponential with reduction since the results obtained by the others exponential expressions diverge. Curves in Fig. 9 show that the curves simulated con- sidering fixed values (reference curves) for displacements $z_{s}$ and $\theta$ is placed between the boundaries curves. So the validity of the calculation method is verified. We notice that the set of the system trajectories have similar look shape as references curves. The displacements $z_{s}$ and $\theta$ are sinusoids that follow the trajectory road.

\section{Dynamic simulation of an active Macpherson suspension system}

Comfort and road handling of a passenger car can be improved by replacing its passive suspension by a controlled suspension. A large number of studies [52-54] exist on the effectiveness of the skyhook control policy along with other optimal control techniques. Most of these studies indicate that skyhook control is the optimal control policy in terms of its ability to isolate the suspended mass from the base excitations. So we implement a skyhook controller in the Macpherson suspension system. One method of generating the skyhook damping force is to remove the passive suspension (i.e., both the damper and the spring) and replace it with an active force generator. This can be achieved by using a hydraulic actuator, however, the resulting system is rather complex and requires a significant amount of power [53]. Another approach to achieving skyhook damping is to use semiactive dampers. Semiactive dampers allow the damping coefficient, and so the damping force, to be varied between high and low levels of damping. Early semiactive dampers were mechanically adjustable by opening or closing a bypass valve. The only power required for the damper is the relatively small power to actuate the valve. Since a semiactive damper is used in this study, we notice that the force applied by the skyhook damper to the suspended mass has the following form

$F_{S K Y}=-C_{S K Y} \cdot z_{S}^{:}$

With $C_{S K Y}$ is the controller damping coefficient.

\subsection{Transient state}

\subsubsection{Fixed values}

Considering the previous matrices except the force $f_{a}$ is nonzero in this case as the suspension system is active. So the force $f_{a}$ is replaced by the relation of $F_{S K Y}$ and the system to solve becomes:

$x^{*}=A x(t)+B_{1}\left(-C_{S K Y} \cdot z_{s}^{*}\right)+B_{2} z_{r}(t)+B_{3} f_{d}(t)$

After substitution of Eq. (37), we obtain the following system:

$x^{*}=A x(t)$ 

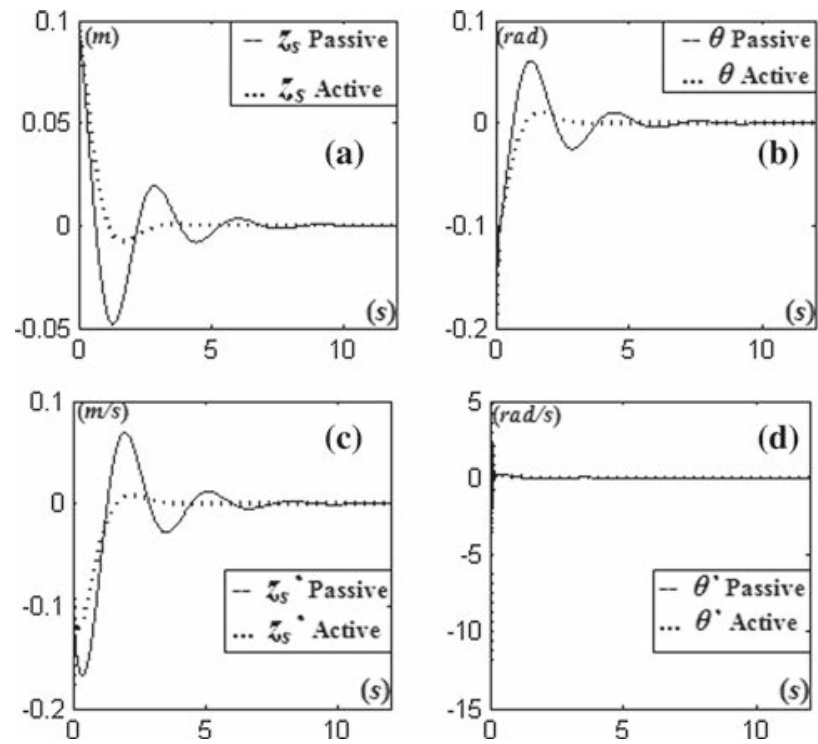

Fig. 10 Comparison between active and passive MacPherson suspension system response when excited with snap-displacement. a Vertical displacement of mass $m_{s}$. b Rotation of mass $m_{s}$ around X. c Linear velocity of mass $m_{s}$. d Angular velocity of mass $m_{s}$

where,

$A=\left[\begin{array}{cccc}0 & 1 & 0 & 0 \\ a_{21} & -C_{S K Y} \frac{l_{B} \cos \left(-\theta_{0}\right)}{m_{s} l_{C}+m_{u} l_{C} \sin ^{2}\left(-\theta_{0}\right)} & a_{23} & a_{24} \\ 0 & 0 & 0 & 1 \\ a_{41} & -C_{S K Y} \frac{\left(m_{s}+m_{u}\right) l_{B}}{m_{s} m_{u} l_{C}^{2}+m_{u}^{2} l_{C}^{2} \sin ^{2}\left(-\theta_{0}\right)} & a_{43} & a_{44}\end{array}\right]$

and

$f_{d}=0$.

After calculation which was done without uncertainty and with controller damping coefficient $C_{S K Y}=800 \mathrm{~N} . \mathrm{s} / \mathrm{m}$ we obtain results in Fig. 10.

Figure 10a, b, c, d show the comparison of step response of MacPherson syspension system with skyhook and without skyhook controller for outputs $z_{s}, z_{s}^{*}, \theta$ and $\theta \cdot$. From Fig. 10 we notice that when we use controller overshot decrease and system reaches to steady state faster. It is noticeable from these figures that the magnitude for the body vehicle decreases even its velocity and the vehicle resumes its equilibrium state in $3 \mathrm{~s}$ instead of $8 \mathrm{~s}$. This is an improvement of the system response which confirm the efficiency of the skyhook controller to the reduce of vibrations caused by the roads. The velocity variation (Fig. 10c, d) of active behaviour of MacPherson suspension is too small compared to the velocity variation of the passive behaviour which reflects that the acceleration of the body vehicle is reduced.

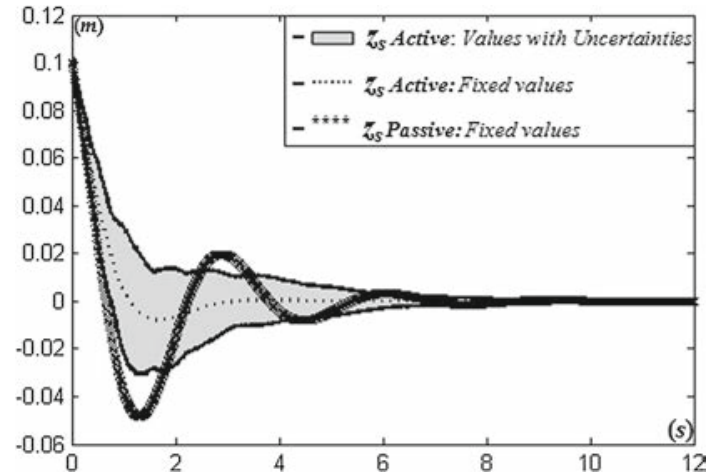

Fig. 11 MacPherson suspension system response when excited with snap-displacement. a Vertical displacement of mass ms calculated by applying Horner exponential with reduction.

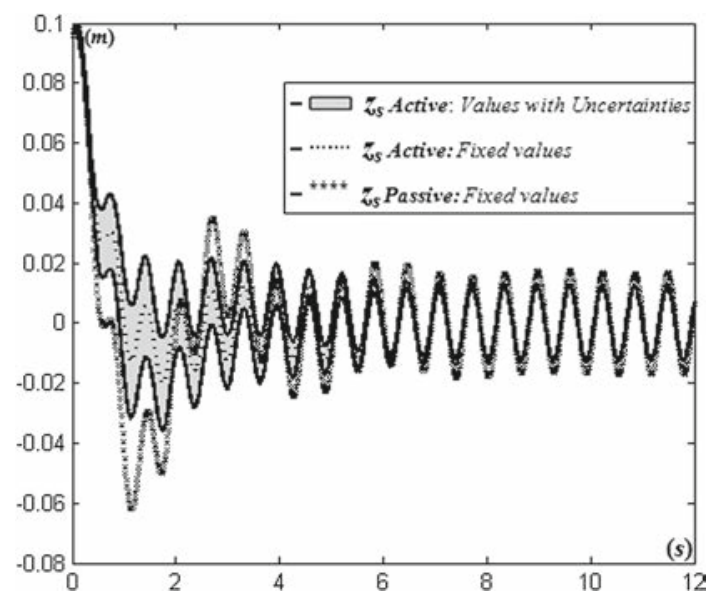

Fig. 12 MacPherson suspension system response when excited with snap-displacement. a Vertical displacement of mass ms calculated by applying Horner exponential with reduction.

\subsubsection{Uncertainties}

The selection of the optimal controller damping coefficient is crucial, since it determines the optimal manner of the MacPherson suspension behaviour. So according to excitation kind, we need to determine if the controller damping coefficient is well chosen or not. So $2 \%$ of uncertainties on the controller damping coefficient $\left(C_{S K Y}=[784 ; 816]\right)$ is introduced and the uncertainties of the values of design parameters is setted to zero.

From curve in Fig. 11 we notice that even if we take a higher percentage of uncertainty on the damping controller coefficient the results obtained converge. This is due to the fact that the uncertainty was implemented in one design variable $C_{S K Y}$.

\subsection{Harmonic state}

With the uncertainties introduced on the damping controller coefficient, the designer can make a good idea on the effect 
of the increasing or the reducing of this coefficient on the behaviour of the MacPherson suspension system. So the simulation with interval can help to make a good decision about the choice of design parameters (Fig. 12).

With the interval based simulation method, several dynamic simulations result from the uncertainty imposed on the variables of the dynamic model. These performance bounds and the dynamic behavior of the system under nominal value can provide more complete information for a designer. So relying on these simulations, the designer can determine the solution that can fulfill the constraints imposed in the specifications document, which makes the sizing of the MacPherson suspension system easier.

\section{Conclusion}

From the previous study of dynamic behavior of the MacPherson suspension system, the validity of interval based simulation to solve differential equations has been proved. With the use of Horner exponential with reduction, the exponential divergence problem when the Interval Based Simulation is applied and solved and the results of simulation are more realistic. However, this type of calculation is orientated to solve linear ordinary differential equations to make dynamic simulations. Indeed, the interval based simulation compared to a classical simulation allows multiple simulations simultaneously and recesses to reduce of the computational burden. The Interval Based Simulation method provides not only a single evaluation of the system behavior but also a set of performance bounds. Simulation results demonstrate that these performance bounds give a better description of the dynamic behavior of MacPherson suspension system with uncertain parameters.

\section{References}

1. Pahl, G., Beitz, W.: Engineering design: a systematic approach, 2nd edn. Springer, London (1996)

2. Roshanian, J., Keshavarz, Z.: Effect of variable selection on multidisciplinary design optimization: a flight vehicle example. Chin. J. Aeronaut. 20(1), 86-96 (2006)

3. Eltimsahy, A.H., Copass, C.H.: Computer simulation for a solar heating system. Math. Comput. Simul. 20(2), 114-127 (1978)

4. Teorey, T.J., Yang, D., Fry, J.P.: A logical design methodology for relational databases using the extended entity relationship Model. N. Y. ACM Comput. Surveys 18(2), 197-222 (1986)

5. Paredes, M., Yang, D., Fry, J.P.: Methodology to build an assistance tool dedicated to preliminary design: application to compression springs. Int. J. Interact. Design Manuf. (IJIDeM) 3(4), 265-272 (2009)

6. Yan, H.S.: A methodology for creative mechanism design. Mech. Machine Theory 27(3), 235-242 (2002)

7. Modelica, modeling of complex physical systems, Modelica. http:// www.modelica.org/

8. Catia, virtual design. http://www.3ds.com/fr/products/catia/ welcome/

9. Cosmosworks. http://www.cosmosworks.com/
10. Wang, Z., Wang, H.: Inflatable wing design parameter optimization using orthogonal testing and support vector machines. Chin. J. Aeronaut. 25(6), 887-895 (2012)

11. Croccolo, D., De Agostinis, M., Vincenzi, N.: Design and optimization of shafthub hybrid joints for lightweight structures: Analytical definition of normalizing parameters. Int. J. Mech. Sci. 56(1), 7785 (2012)

12. Telerman, V.: Propagation of mathematical constraints in subdefinite models. In: Calmet, J., Campbell J.A. (Eds.) Integrating symbolic mathematical computation and artificial intelligence, LNCS. 958:191-208 (1995)

13. Shvetsov, I., Telerman, V., NeMo+, D.: Ushakov, object-oriented constraint programming environment based on subdefinite models. In: Gert Smolka (Ed.) Principles and practice of constraint programming - CP97. Proceedings. LNCS, 1330, 534-548 (1997)

14. Moore, R.E.: Interval analysis. Prentice-Hall, New Jersey (1966). ISBN 0-13-476853-1

15. Jaulin, L., Kieffer, M., Didrit, O., Walter, E.: Applied interval analysis. Springer, New York (2001)

16. Granvilliers, L., Monfroy, E., Benhamou, F.: Symbolic-interval cooperation in constraint programming. ISSAC ' 01 Proceedings of the 2001 international symposium on symbolic and algebraic computation. 150-166 (2001)

17. Eldon, H.: Global optimization using interval analysis: the one-dimensional case. J. Optim. Theory Appl. 29(4), 331-344 (2002)

18. Revol, N.: Arithmétique par intervalles, Calculateurs Parallèles et Systèmes Rèpartis, vol. 13, pp. 387-426 (2001) (in french)

19. Meslem, N., Ramdani, N., Candau, Y.: Guaranteed approximation of reachable sets for uncertain dynamical continuous-time systems. Eur. J. Autom. Systems 43(6), 241-266 (2009)

20. Croisard, N., Vasile, M., Kemble, S., Radice, G.: Preliminary space mission design under uncertainty. Acta Astronautica 66(5-6), 654664 (2010)

21. Cabannes, G., Troussier, N., Gidel, T., Cherfi, Z.: An uncertaintybased approach to drive product preliminary design. Int. J. Interact. Design Manuf (IJIDeM) 5(1), 55-65 (2011)

22. Montanari, U.: Networks of constraints: fundamental properties and application to picture processing. Inf. Sci. 7, 95-132 (1974)

23. Brailsford, S.C., Potts, C.N., Smith, B.M.: Constraint satisfaction problems: algorithms and applications. Eur. J. Oper. Res. 119(3), 557-581 (1999)

24. Yvars, P.A.: Using constraint satisfaction for designing mechanical systems. Int. J. Interact. Design Manuf. (IJIDeM) 2(3), 161-167 (2008)

25. Yvars, P.A., Lafon, P., Zimmer, L.: Optimization of mechanical system: contribution of constraint satisfaction method. proc of IEEE International Conference of Computers and industrial engineering, CIE'39, Troyes, 1379-1384 (2009)

26. Fischer, X.: Strategie de conduite du calcul pour l'aide la decision en conception integree; application aux appareils a pression, ENSAM, Phd Thesis (2000)

27. Bensana, E., Mulyanto, T.: A generic approach for conceptual design based on object oriented and constraint logic programming. In: International conference on engineering design and automation, Orlando, Floride (2000)

28. Scaravetti, D., Nadeau, J.P., Pailhs, J., Sebastian, P.: Structuring of embodiment design problem based on the product lifecycle. Int. J. Product Dev. 2, N1-2 (2005)

29. Chenouard, R., Granvilliers, L., Sbastian, P.: Search heuristics for constraint-aided embodiment design. Artif. Intell. Eng. Design Analysis Manuf. (AIEDAM) 23(2), 175-195 (2009)

30. Vargas, C., Saucier, A., Yvars, P.A.: An environment for mechnical design aid applications development, Revue Internationale de CFAO et d'Infographie, Vol 10 - N1-2. Herms, France (1995) 
31. Sellini, F., Yvars, P.A.: Veri-KoMoD : Verification of knowledge models in the mechanical design field, knowledge acquisition, modeling and management. Lecture notes in computer science, ed. 1621, pp. 385-390. Springer (1999)

32. Yannou, B., Harmel, G.: A comparative study of constraint programming techniques over intervals in preliminary design. In: Proceedings of ASME Design Engineering Technical Conferences. ASME, 189198 (2004)

33. Aldanondo, M., Hadj-Hamou, K., Moynard, G., Lamothe, J.: Mass customization and configuration: requirement analysis and constraint based modeling propositions. Integr. Comput. Aided Eng. 10(2), 177-189 (2003)

34. Larroud, V., Chenouard, R., Yvars, P.A., Millet, D.: Constraint based approach for the steady-state simulation of complex systems : application to ship control. Eng. Appl. Artif. Intell. 26(1), 499514 (2013)

35. Tcherctchian, N., Yvars, P.A., Millet, D.: Benefits and limits of a csp/lca approach for the ecodesign of complex systems. a case applied to a hybrid passenger ferry. J. Cleaner Prod. 42, 118 (2013)

36. Canbaz, B., Yannou, B, Yvars P.A.: A multiplayer simulation of a set-based design framework with CSP techniques, proc of IDETC/CIE 2012 (2012)

37. Ward, R.C.: Numerical computation of the matrix exponential with accuracy estimate. SIAM J. Numer. Analysis 14(4), 600-610 (1977)

38. Bochev, P., Markov, S.: A self-validating numerical method for the matrix exponential. Computing 43(1), 59-72 (1989)

39. Moler, C., Loan, C.V.: Nineteen dubious ways to compute the exponential of a matrix. Soc. Ind. Appl. Math. Review 45(1), 3-49 (2003)

40. Goldsztejn, A.: On the exponentiation of interval matrices, Hal00411330. http://hal.archivesouvertes.fr/docs/00/41/13/30/PDF/ IntervalMatrixExponential.pdf. August 2009

41. Goldsztejn, A., Mullier, O., Eveillard, D., Hosobe, H.: Including ordinary difierential equations based constraints in the standard CP frame work, CP' 10 proceedings of the 16th international conference on principles and practice of constraint programming. 221$235(2010)$

42. Rump, S.M.: S.M. INTLAB - INTerval LABoratory. In: Tibor Csendes, editor, Developments in reliable computing, pp. 77104. Kluwer Academic Publishers, Dordrecht. http://www.ti3. tu-harburg.de/rump/ (1999)
43. Oppenheimer, E.P., Michel, A.N.: Application of interval analysis techniques to linear systems. I. Fundamental results. IEEE Trans. Circuits Systems 632 35(9), 1129-1138 (1988)

44. Oppenheimer, E.P.: Application of interval analysis techniques to linear Sys II. The interval matrix exponential function. IEEE Trans. Circuits Systems 35(10), 1230-1242 (1988)

45. Oppenheimer, E.P., Michel, A.N.: Application of interval analysis techniques to linear system. III. IEEE Trans Initial Value Problems Circuits Systems 35(10), 1243-1256 (1988)

46. Ceberio, M., Granvilliers, L.: Horners rule for interval evaluation revisited. Computing. 69(1), 51-81 (2002)

47. Harris, W.: How car suspensions work: How car suspensions work. How stuff works. http://auto.howstuffworks.com/car-suspension. htm (2005)

48. http://www.raw4x4europe.com/catalogo_en.htm. Accessed 02 Feb 2014

49. Habibi, H., Shirazi, K.H., Shishesaz, M.: Roll steer minimization of McPherson-strut suspension system using genetic algorithm method. Mech. Machine Theory 43(1), 57-67 (2008)

50. Papegay, Y.A., Merlet, J.P., Daney, D.: Exact kinematics analysis of Cars suspension mechanisms using symbolic computation and interval analysis. Mech. Machine Theory 40(4), 395-413 (2007)

51. Hong, K.S., Jeon, D.S., Sohn, H.C.: A new modeling of the Macpherson suspension system and its optimal pole-placement control. Proceedings of the 7th Mediterranean conference on control and automation (MED99) Haifa, Israel, June 28-30 (1999)

52. Li, H., Goodall, R.M.: Linear and non-linear skyhook damping control laws for active railway suspensions. Control Eng. Pract. 7(7), 843-850 (1999)

53. Priyandoko, G., Mailah, M., Jamaluddin, H.: Vehicle active suspension system using skyhook adaptive neuro active force control. Mech. Systems Signal Process. 23(3), 855-868 (2009)

54. Choi, S.B., Lee, H.K., Chang, E.G.: Field test results of a semiactive ER suspension system associated with skyhook controller. Mechatronics 11(3), 345-353 (2001) 